\title{
Gas Sensing Properties of Graphene-Rb-Based Sensor for Liquefied Petroleum Gas and Hydrogen
}

\author{
Shivani A. Singh, Pravin. S. More* \\ Nano Material Application Lab., Department of Physics, Institute of Science, Madam Kama Road, Fort, Mumbai, \\ Maharashtra, India \\ *Corresponding author Phone: 8356002365; E-mail: shivani.singh@tcetmumbai.in, \\ shivani28593@gmail.com
}

\begin{abstract}
Article Info

Volume 8, Issue 3

Page Number : 353-359

\section{Publication Issue}

May-June-2021

\section{Article History}

Accepted : 20 May 2021

Published : 25 May 2021

$\mathrm{Rb}$-modified graphene powder was prepared by the chemical route synthesis method in this paper. X-ray powder diffraction (XRD) and scanning electron microscopy (SEM) were used to characterize the composition of the crystalline phase and the morphology of the prepared gas-sensitive materials, respectively. In particular, the study focused on the sensing behaviours of Graphene-Rb-based sensor towards power transformer fault gases such as hydrogen and liquefied petroleum gas. The molar concentration (M) level of $\mathrm{Rb}$ additive was varied systematically from $0.5 \mathrm{M}$ to $2.0 \mathrm{M}$. The highest value of sensitivity factor (SF) of 200 for 1.0M Rb-modified graphene to LPG gas was obtained at considerably lower temperature of 390K and for Hydrogen gas the highest value of sensitivity factor (SF) of $\sim 225$ for $1.0 \mathrm{M} \mathrm{Rb}$-modified graphene at considerably lower temperature of $380 \mathrm{~K}$. The selectivity value is found to be maximum 16 for 1.5M of Rb-modified graphene for LPG and 32 for 2.0M of Rb-modified graphene for Hydrogen gas.
\end{abstract}

Keywords : LPG Sensor; Hydrogen Sensor; Rb-modified Graphene; Sensitivity; Selectivity.

\section{INTRODUCTION}

Carbon the 6th element in the periodic tables has always remains a fascinating material to the researcher and technologist. Diamond, graphite, fullerenes, carbon nanotubes and newly discovered graphene are the most studied allotropes of the carbon family. The significance of these material can be understood as the discovery of fullerene and graphene has been awarded noble prizes in the years 1996 and 2010 to Curl, Kroto \& Smalley and Geim \& Novalec, respectively. After the flood of publications on graphite intercalated [1], fullerenes (1985) [2], and carbon nanotubes (1991) [3], graphene have been the 
subject of countless publications since $2004[4,5]$. Graphene is a flat monolayer of carbon atoms tightly packed into a two-dimensional (2D) Honeycomb lattice; completely conjugated $\mathrm{sp}^{2}$ hybridized planar structure and is a basic building block for graphitic materials of all other dimensionalities (Figure 1). It can be wrapped up into 0D fullerenes, rolled into 1D nanotube or stacked into 3D graphite.

In 2004, Geim and co-workers at Manchester University successfully identified single layers of graphene in a simple tabletop experiment and added a revolutionary discovery in the field of nano science and nanotechnology. Interest in graphene increased dramatically after Novoselov, Geim et al. reported on the unusual electronic properties of single layers of the graphite lattice. One of the most remarkable properties of graphene is that its charge carriers behave as massless relativistic particles or Dirac fermions, and under ambient conditions they can move with little scattering. This unique behavior has led to a number of exceptional.

Phenomena in graphene [4]. First, graphene is a zeroband gap 2D semiconductor with a tiny overlap between valence and conduction bands. Second, it exhibits a strong ambipolar electric field effect so that the charge carrier concentrations of up to $1013 \mathrm{~cm}^{-2}$ and room-temperature mobility of $10000 \mathrm{~cm}^{-2} \mathrm{~s}^{-1}$ are measured. Third, an unusual half-integer quantum Hall effect (QHE) for both electron and hole carriers in graphene has been observed by adjusting the chemical potential using the electric field effect $[5,6]$. It has high thermal conductivity with a value of 5000 $\mathrm{WmK}^{-1}$ for a single-layer sheet at room temperature. In addition, graphene is highly transparent, with absorption of $2.3 \%$ towards visible light $[7,8]$. Narrow ribbons of graphene with a thickness of 1-2 $\mathrm{nm}$ are, however, semiconductors with a distinct band gap and these can be used to produce transistors [9-11].
In last couples of years, graphene has been used as alternative carbon-based nanofiller in the preparation of polymer nanocomposites and have shown improved mechanical, thermal, and electrical properties [12-19]. The recent advances have shown that it can replace brittle and chemically unstable indium tin oxide in flexible displays and touch screens [20-21]. It is well established that the superior properties of graphene are associated with its singlelayer. However, the fabrication of single-layer graphene is difficult at ambient temperature. If the sheets are not well separated from each other than graphene sheets with a high surface area tend to form irreversible agglomerates and restacks to form graphite through $\mathrm{p}-\mathrm{p}$ stacking and Vander Waals interactions [22, 23]. Aggregation can be reduced by the attachment of other small molecules or polymers to the graphene sheets. The presence of hydrophilic or hydrophobic groups prevents aggregation of graphene sheets by strong polar-polar interactions or by their bulky size [24]. The attachment of functional groups to graphene also aids in dispersion in a hydrophilic or hydrophobic media, as well as in the organic polymer.

Therefore, an efficient approach to the production of surface-functionalized graphene sheets in large quantities has been a major focus of many researchers. The goal is to exploit the most frequently proposed applications of graphene in the areas of polymer nanocomposites, super-capacitor devices, drug delivery systems, solar cells, memory devices, transistor devices, biosensors and electromagnetic/ microwave absorption shields. Before submitting your final paper, check that the format conforms to this template. Specifically, check the appearance of the title and author block, the appearance of section headings, document margins, column width, column spacing and other features. 


\section{METHODS AND MATERIAL}

$\mathrm{Rb}$-modified graphene is synthesized by chemical route method and the synthesized material was used for LPG and Hydrogen gas sensing application. To prepare $\mathrm{Rb}$-modified graphene, rubidium chloride ( $\mathrm{RbCl}$ ), graphite (particle size $40 \mu \mathrm{m}$ ), conc. sulphuric acid $\left(\mathrm{H}_{2} \mathrm{SO}_{4}, 98 \%\right)$, sodium nitrate $\left(\mathrm{NaNO}_{3}\right)$, potassium permanganate $\left(\mathrm{KMnO}_{4}\right)$, and hydrogen peroxide $\left(\mathrm{H}_{2} \mathrm{O}_{2}, 30\right.$ wt. \%) were obtained from S.D.fine Chem. Graphite, $\mathrm{NaNO}_{3}$, and $\mathrm{KMnO}_{4}$ were used as obtained. Deionized water was used in the synthesis to ensure high purity of sample.

Structural and physiochemical properties of the resulting pellets were explored with the help of X-ray powder diffraction (XRD) and scanning electron microscopy (SEM) and gas sensing characteristics by four probe analysis. The Surface morphology of the synthesized products was observed using scanning electron microscopy (SEM) (JEOL, JSM-IT300). For the identification of crystal phase and to calculate various structural parameters of the obtained samples, $\mathrm{X}$-ray diffraction (XRD) pattern were recorded using XPERT-PROMPD X-ray diffractometer, with $\mathrm{CuK} \alpha$ radiation $(\lambda=1.5405 \AA)$ in the $2 \theta$ range of $10-60$. Gas sensing eexperiments were carried out in a sealed chamber. To measure conductivity, a Keithley 2400 source meter was used.

\section{III.RESULTS AND DISCUSSION}

To characterize the performance of the fabricated Graphene-Rb-Based Sensor device as a gas sensor, we measured the change in its resistance upon exposure to varying relative temperature of the sample inside the chamber with different molar concentration of $\mathrm{Rb}$ from $0.5 \mathrm{M}$ to $3.0 \mathrm{M}$. Figure $1(\mathrm{a})$ and (b) shows variations of sensitivity with the temperature at different concentration levels of $\mathrm{Rb}$-modified graphene for different molar concentration for LPG and $\mathrm{H}_{2}$ gas (at $1000 \mathrm{ppm}$ ). It is clear that the SF is maximum of $\sim 200$ for 1.0M Rb-modified graphene to LPG gas was obtained at considerably lower temperature of $390 \mathrm{~K}$ and for Hydrogen gas the highest value of sensitivity factor (SF) of $\sim 225$ for $1.0 \mathrm{M} \mathrm{Rb}$-modified graphene at considerably lower temperature of $380 \mathrm{~K}$. The gas sensing response shows good dependence on the LPG and $\mathrm{H}_{2}$ concentration as compared to other $\mathrm{Rb}$ concentration.
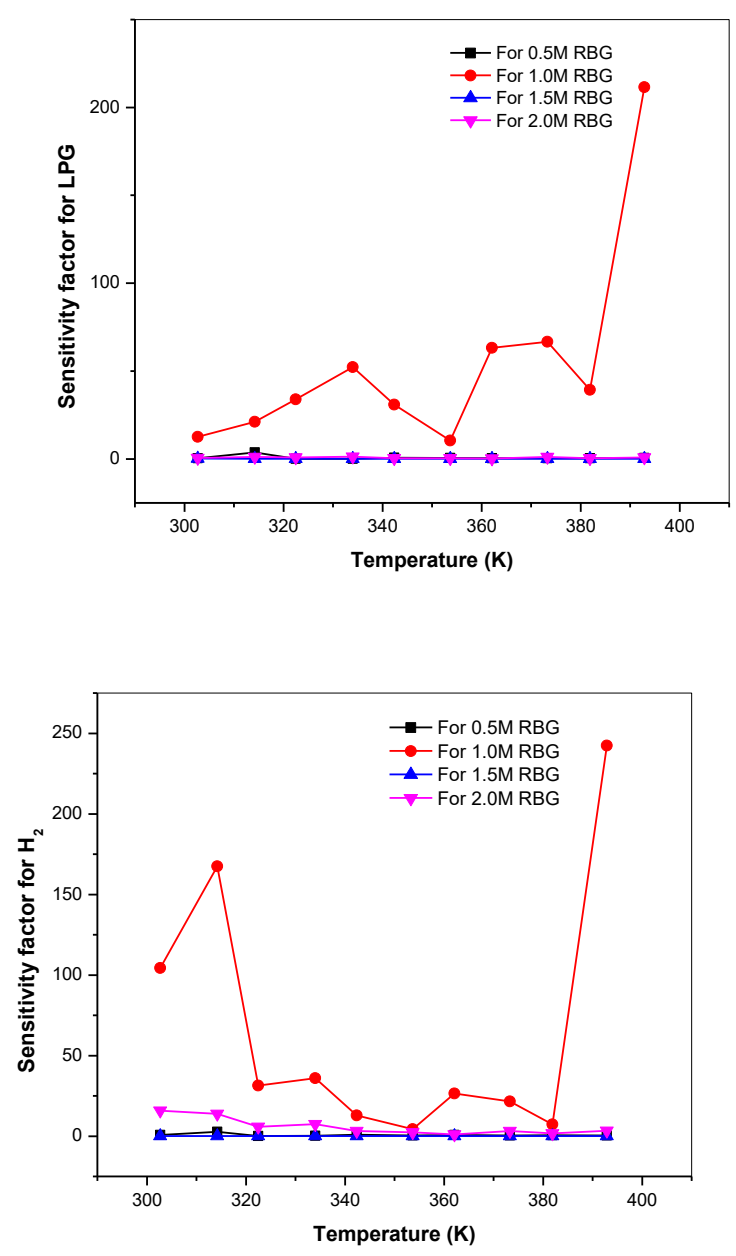

Figure 1 (a) variation SF with operating temperature for different molar concentration of $\mathrm{Rb}$ (at $1000 \mathrm{ppm}$ of LPG).

(b) Variation SF with operating temperature for different molar concentration of $\mathrm{Rb}$ (at $1000 \mathrm{ppm}$ of $\mathrm{H}_{2}$ ).

The variation of selectivity with $\mathrm{Rb}$ molar concentration LPG and $\mathrm{H}_{2}$. Here, the selectivity is defined as the ratio of SF for test gas and interfering 
gases (at the same concentration of $1000 \mathrm{ppm}$ ) at the optimized operating temperature for the test gas. The optimum concentrations of Rb-modified graphene as regards the selectivity for LPG are found to be maximum 16 for $1.5 \mathrm{M}$ of $\mathrm{Rb}$-modified graphene for LPG and 32 for $2.0 \mathrm{M}$ of Rb-modified graphene for Hydrogen gas as shown in figure 2 (a) and (b).
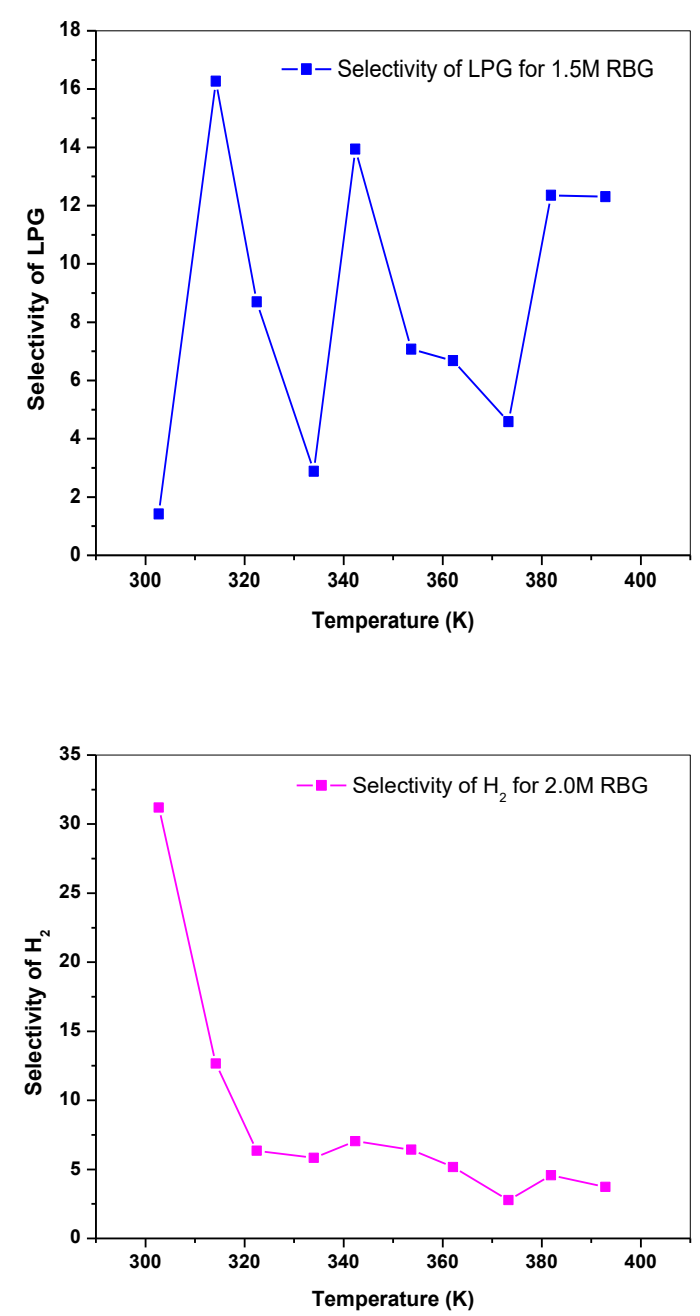

Figure 2 (a) Selectivity variation at different molar concentration of $\mathrm{Rb}$ (at $1000 \mathrm{ppm}$ of LPG). (b) Selectivity variation at different molar concentration of $\mathrm{Rb}$ (at $1000 \mathrm{ppm}$ of $\mathrm{H}_{2}$ ).

The XRD pattern of pure graphene particles (Fig. 3) shows that the characteristic peak of $\mathrm{Rb}$-modified graphene is at $26.6^{\circ}$. Figure 3 shows the $\mathrm{XRD}$ pattern of Graphene and $\mathrm{Rb}^{-}$modified graphene nanoparticles at different molar concentration of $\mathrm{Rb}$. The peak position of all the XRD pattern is in good agreement The XRD studies were carried out to understand the crystallinity and phase of the samples, respectively. The XRD pattern reveals that the Graphene nanoparticles are more amorphous in nature were with doping of $\mathrm{Rb}$ it shoes crystalline nature.

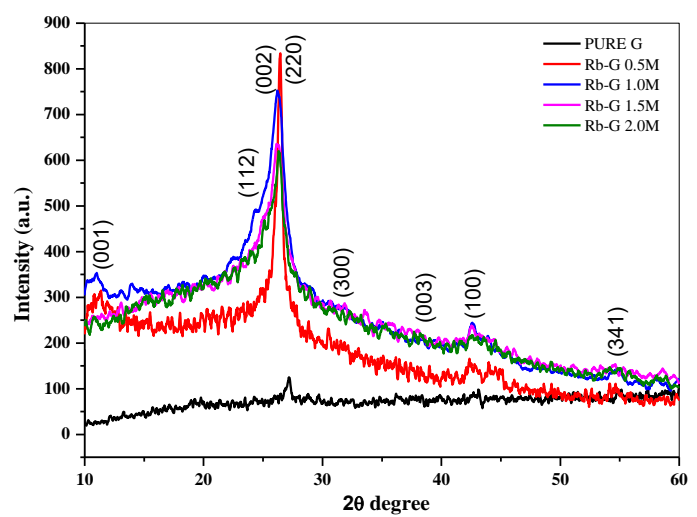

Figure 3. XRD pattern of Graphene and Rb- modified graphene nanoparticles at different molar concentration of $\mathrm{Rb}$.

SEM images of the Graphene and $\mathrm{Rb}^{-}$modified graphene nanoparticles are shown in Figure 4(a), 4(b), 4(c), 4(d) and 4(e) for pure graphene and different molar concentration of $\mathrm{Rb}$-doping such as $0.5 \mathrm{M}, 1.0 \mathrm{M}$, $1.5 \mathrm{M}$ and $2.0 \mathrm{M}$ respectively. It is seen that the morphology of nanostructures strongly depends on the molar concentration of Rb. In Fig. 4 (a) the SEM image of highly oxidized graphite shows a smooth surface with tightly packed sheets, while the exfoliated sample exists as transparent, wrinkled sheets, indicating these layers are exfoliated to a very large extent. They are well organized. In the further SEM images 4(b), 4(c), $4(\mathrm{~d})$ and $4(\mathrm{e})$ it is clearly seen that by doping of $\mathrm{Rb}$ the morphology changes from wrinkled sheets to granular like structure. Increasing molar concentration of $\mathrm{Rb}$ can cause further change in the morphology of samples. 

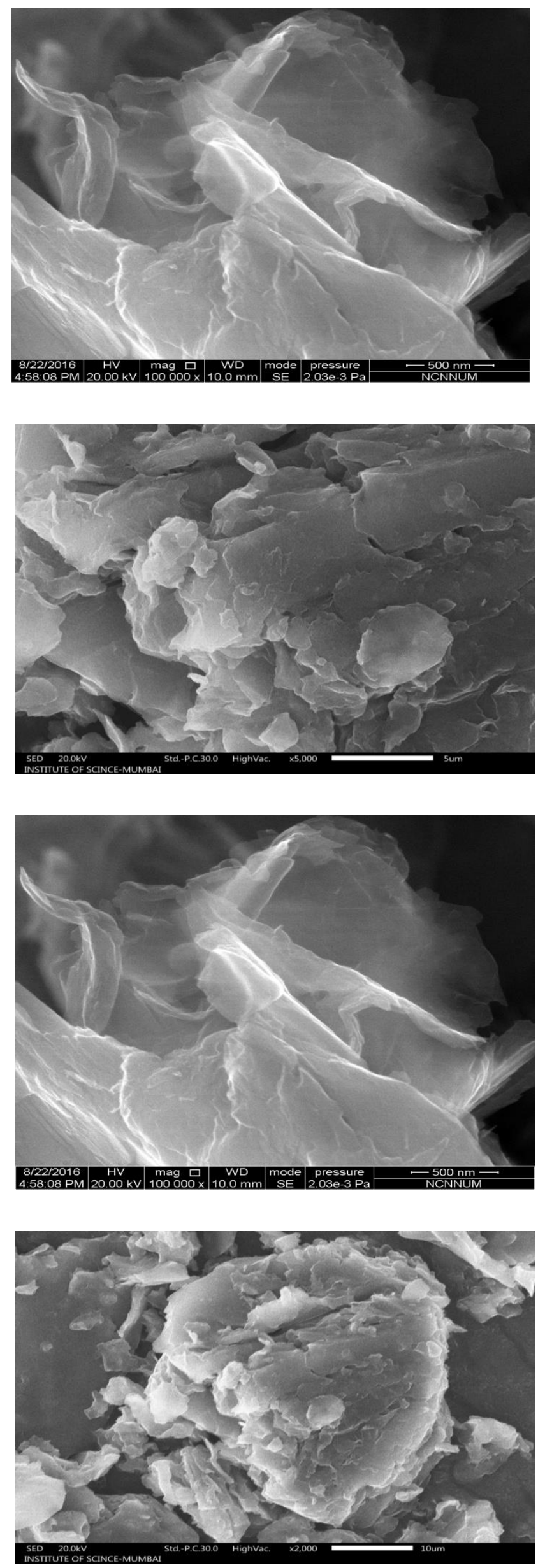

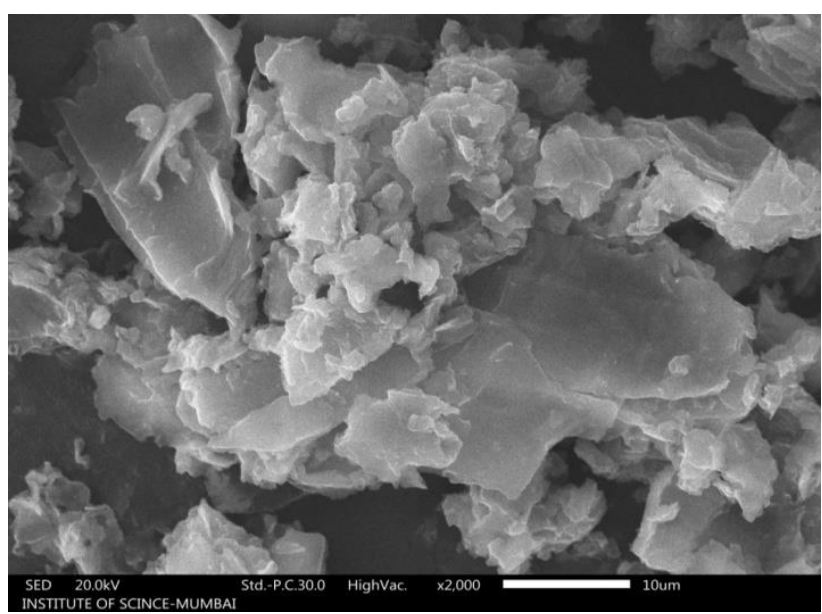

Figure 4. SEM images of the Graphene and Rbmodified graphene nanoparticles

\section{CONCLUSION}

In the summary, we successfully demonstrated LPG and $\mathrm{H}_{2}$ sensing application by $\mathrm{Rb}$-modified graphene composite. The as-prepared sample shows good sensing and operating temperature response. The sample shows good selectivity characteristics. These high sensitivity and wider range characteristics are achieved by using considerably lower concentration level of $\mathrm{Rb}$ additive as compared to the reported data. The main achievement of present work is low operating temperature, which is much below autoignition temperature of LPG and $\mathrm{H}_{2}$. The graphene sensor shows significant conductance changes when exposed to various concentrations of testing gasses in air. The response time of the sensor is less than $10 \mathrm{~s}$, suggesting that the device shows fast response to LPG and $\mathrm{H}_{2}$ gas. Because of the weak interaction between gases and graphene, the device response is rapid and reproducible. In the future, we will investigate the feasibility of using graphene sensors for environmental monitoring and carry out surface fictionalization of the graphene layers to enhance the selectivity of the sensor. 


\section{ACKNOLEGMENT}

One of the authors P. S. More is thankful to University Grant Commission (UGC), New Delhi, and Director, Higher and Technical Education, Pune, India for financial support.

\section{REFERENCES}

[1]. Lincoln Vogel, F. (1977). The electrical conductivity of graphite intercalated with superacid fluorides: experiments with antimony pentafluoride. Journal of Materials Science, 12(5), 982-986.

[2]. Kroto, H. W., Heath, J. R., O’Brien, S. C., Curl, R. F., \& Smalley, R. E. (1985). C60: Buckminsterfullerene. Nature, 318(6042), 162163.

[3]. Iijima, S. (1991). Helical microtubules of graphitic carbon. Nature, 354(6348), 56-58.

[4]. Novoselov, K.S., Geim, A.K., Morozov, S.V., Jiang, D., Zhang, Y., Dubonos, S.V., Grigorieva, I.V., \& Firsov, A.A. (2004). Electric Field Effect in Atomically Thin Carbon Films. Science, 306(5696), 666-669.

[5]. Novoselov, A. K., Geim, S. V., Morozov, D., Jiang, M. I., Katsnelson, I.V., Grigorieva, S. V., Dubonos, S. V., \& Firsov, A. A. (2005). Twodimensional gas of massless Dirac fermions in graphene. Nature, 438(7065), 197-200.

[6]. Zhang, Y., Tan, Y.W., Stormer, H.L., \& Kim, P. (2005). Experimental observation of the quantum Hall effect and Berry's phase in graphene. Nature, 438(7065), 201-204.

[7]. Balandin, A. A., Ghosh, S., Bao, W., Calizo, I., Teweldebrhan, D., Miao, F., \& Lau, C. N. (2008). Superior Thermal Conductivity of Single-Layer Graphene. Nano Letters, 8(3), 902907.

[8]. Nair, R. R., Blake, P., Grigorenko, A. N., Novoselov, K. S., Booth, T. J., Stauber, T., Peres, N. M. R., \& Geim, A. K. (2008). Fine Structure
Constant Defines Visual Transparency of Graphene. Science, 320(5881), 1308.

[9]. Li, X., Wang, X., Zhang, L., Lee, S., \& Dai, H. (2008). Chemically Derived, Ultrasmooth Graphene Nanoribbon Semiconductors. Science, 319(5867), 1229-1232.

[10]. Ritter, K. A., \& Lyding, J. W. (2009). The influence of edge structure on the electronic properties of graphene quantum dots and nanoribbons. Nat Mater, 8(3), 235-242. 60 Nanocomposites - New Trends and Developments

[11]. Cai, J., Ruffieux, P., Jaafar, R., Bieri, M., Braun, T., Blankenburg, S., Muoth, M., Seitsonen, A. P., Saleh, M., Feng, X., Mullen, K., \& Fasel, R. (2010). Atomically precise bottom- up fabrication of graphene nanoribbons. Nature, 466(7305), 470-473.

[12]. Ansari, S., \& Giannelis, E. P. (2009). Functionalized graphene sheet-Poly(vinylidene fluoride) conductive nanocomposites. Journal of Polymer Science Part B: Polymer Physics, 47(9), 888-897.

[13]. Ramanathan, T., Abdala, A. A., Stankovich, S., Dikin, D. A., Herrera, Alonso. M., Piner, R. D., Adamson, D. H., Schniepp, H. C., Chen, X., Ruoff, R. S., Nguyen, S. T., Aksay, I. A., Prud'Homme, R. K., \& Brinson, L. C. (2008). Functionalized graphene sheets for polymer nanocomposites. Nat Nano, 3(6), 327-331.

[14]. Stankovich, S., Dikin, D. A., Dommett, G. H. B., Kohlhaas, K. M., Zimney, E. J., Stach, E. A., Piner, R. D., Nguyen, S. T., \& Ruoff, R. S. (2006). Graphene-based composite materials. Nature, 442(7100), 282-286.

[15]. Fan, H., Wang, L., Zhao, K., Li, N., Shi, Z., Ge, Z., \& Jin, Z. (2010). Fabrication, Mechanical Properties, and Biocompatibility of GrapheneReinforced Chitosan Composites. Biomacromolecules, 11(9), 2345-2351.

[16]. Zhang, K., Zhang, L. L., Zhao, X. S., \& Wu, J. (2010). Graphene/Polyaniline Nanofiber 
Composites as Supercapacitor Electrodes. Chemistry of Materials. ; , 22(4), 1392-1401.

[17]. Zhao, X., Zhang, Q., Chen, D., \& Lu, P. (2010). Enhanced Mechanical Properties of GrapheneBased Poly(vinyl alcohol) Composites. Macromolecules, 43(5), 2357-2363.

[18]. Kuila, T., Bose, S., Hong, C. E., Uddin, M. E., Khanra, P., Kim, N. H., \& Lee, J. H. (2011). Preparation of functionalized graphene/linear low density polyethylene composites by a solution mixing method. Carbon, 49(3), 10331037.

[19]. Wu, J., Becerril, H. A., Bao, Z., Liu, Z., Chen, Y., \& Peumans, P. (2008). Organic solar cells with solution-processed graphene transparent electrodes. Applied Physics Letters, 92(26), 263302.

[20]. Huang, J., Wang, X., de Mello, A. J., de Mello, J. C., \& Bradley, D. D. C. (2007). Efficient flexible polymer light emitting diodes with conducting polymer anodes. Journal of Materials Chemistry, 17(33), 3551-3554.

[21]. Park, H., Rowehl, J. A., Kim, K. K., Bulovic, V., \& Kong, J. (2010). Doped graphene electrodes for organic solar cells. Nanotechnology, 21(505204).

[22]. Li, D., Muller, M. B., Gilje, S., Kaner, R. B., \& Wallace, G. G. (2008). Processable aqueous dispersions of graphene nanosheets. Nat Nano, 3(2), 101-105.

[23]. Shan, C., Yang, H., Han, D., Zhang, Q., Ivaska, A., \& Niu, L. (2009). Water-Soluble Graphene Covalently Functionalized by Biocompatible Poly-l-lysine. Langmuir, 25(20), 12030-12033.

[24]. Si, Y., \& Samulski, E. T. (2008). Synthesis of Water Soluble Graphene. Nano Letters, 8(6), 1679-1682.

\section{Cite this article as :}

Shivani A. Singh, Pravin. S. More, "Gas Sensing Properties of Graphene-Rb-Based Sensor for Liquefied Petroleum Gas and Hydrogen", International Journal of Scientific Research in Science and Technology (IJSRST), Online ISSN : 2395-602X, Print ISSN : 2395-6011, Volume 8 Issue 3, pp. 353-359, May-June 2021. Available at doi : https://doi.org/10.32628/IJSRST218374 Journal URL : https://ijsrst.com/IJSRST218374 